\title{
Neutrophil:lymphocyte ratio is positively related to type 2 diabetes in a large-scale adult population: a Tianjin Chronic Low-Grade Systemic Inflammation and Health cohort study
}

\author{
Xiaoyan Guo ${ }^{1, *}$, Shu Zhang ${ }^{1{ }^{*}}$, Qing Zhang ${ }^{2}$, Li Liu ${ }^{2}$, Hongmei Wu' ${ }^{1}$, Huanmin Du', \\ Hongbin Shi ${ }^{1}$, Chongjin Wang ${ }^{2}$, Yang Xia ${ }^{1}$, Xing Liu' ${ }^{1}$, Chunlei Li ${ }^{1}$, Shaomei Sun ${ }^{2}$, \\ Xing Wang ${ }^{2}$, Ming Zhou ${ }^{2}$, Guowei Huang ${ }^{1}$, Qiyu Jia ${ }^{2}$, Honglin Zhao', Kun Song ${ }^{2}$ \\ and Kaijun Niu' \\ ${ }^{1}$ Nutritional Epidemiology Institute and School of Public Health, Tianjin Medical University, \\ 22 Qixiangtai Road, Heping District, Tianjin 300070, China and ${ }^{2}$ Health Management Centre, \\ Tianjin Medical University General Hospital, Tianjin 300052, China \\ *(X Guo and S Zhang contributed equally to this work)
}

\author{
Correspondence \\ should be addressed \\ to $\mathrm{K} \mathrm{Niu}$ \\ Email \\ niukaijun@tmu.edu.cn or \\ nkj0809@gmail.com
}

\begin{abstract}
Aim: It is widely known that inflammation is related to type 2 diabetes (T2D), but few studies have shown a direct relationship between the immune system and T2D using a reliable biomarker. Neutrophil:lymphocyte ratio (NLR) is an easy-to-analyze inflammation biomarker, but few studies have assessed the relationship between NLR and T2D. In order to evaluate how NLR is related to T2D, we designed a large-scale cross-sectional and prospective cohort study in an adult population.

Subjects and methods: Participants were recruited from the Tianjin Medical University General Hospital-Health Management Centre. Both a baseline cross-sectional $(n=87686)$ and a prospective $(n=38074)$ assessment were performed. Participants without a history of T2D were followed up for $\sim 6$ years (with a median follow-up of 2.7 years). Adjusted logistic and Cox proportional hazards regression models were used to assess relationships between the quintiles of NLR and T2D (covariates: age, sex, BMI, smoking status, drinking status, hypertension, hyperlipidemia, and family history of cardiovascular disease, hypertension, hyperlipidemia, or diabetes).

Results: The prevalence and incidence of T2D were $4.9 \%$ and 6.8/1000 person-years respectively. The adjusted odds ratio and hazard ratio $(95 \% \mathrm{Cl})$ of the highest NLR quintile were $1.34(1.21,1.49)$ and $1.39(1.09,1.78)$ (both $P$ for trend <0.01) respectively as compared to the lowest quintile of NLR. Leukocyte, neutrophil, and lymphocyte counts do not significantly predict the eventual development of T2D.

Conclusion: The present study demonstrates that NLR is related to the prevalence and incidence of T2D, and it suggests that NLR may be an efficient and accurate prognostic biomarker for T2D.

\section{Introduction}

Type 2 diabetes (T2D) is a major global health problem that affects more than 285 million individuals worldwide (1), and it has reached epidemic proportions in China.
An estimated 92.4 million adults age 20 years or older (9.7\% of the adult population) have diabetes, and $60.7 \%$ of these cases are undiagnosed (2). Another 148.2 million (c) 2015 European Society of Endocrinology Printed in Great Britain
Published by Bioscientifica Ltd. 
adults (15.5\%) have pre-diabetes, which is an important risk factor for the development of overt diabetes as well as cardiovascular disease (CVD) $(3,4)$. The increasing burden of T2D (5) demonstrates an immediate need for elucidating the mechanisms that underlie its pathophysiology of in order to implement preventative strategies.

Prior studies have indicated that components of the immune system are altered in obesity and T2D (6) and that elevated levels of the inflammatory markers interleukin 6 (IL6) and C-reactive protein (CRP) are associated with the development of T2D (7). Other research has indicated that inflammation of pancreatic islets can reduce insulin secretion and trigger $\beta$-cell apoptosis (8). This led us to hypothesize that immune markers might be useful predictors of T2D.

Neutrophil:lymphocyte ratio (NLR), which has become one of the most popular biomarkers in biological and medical research in recent years, has been shown to correspond to numerous chronic inflammatory diseases $(9,10)$. NLR is both accessible and affordable, and it thus has been increasingly used in clinical trials and research studies; however, few studies have examined the relationship between NLR and T2D (11), and whether NLR is associated with T2D is unknown. In the present study, we used a cross-sectional and prospective cohort study to evaluate the predictive power of NLR for T2D.

\section{Subjects and methods}

\section{Participants}

The Tianjin Chronic Low-Grade Systemic Inflammation and Health (TCLSIHealth) cohort study is a large prospective dynamic cohort study that focuses on the relationship between chronic low-grade systemic inflammation and the health status of a population that lives in Tianjin, China $(12,13)$. Tianjin is a city of $\sim 10.43$ million inhabitants that is located in the northeast of the North China Plain and faces the Bohai Sea (14). Participants were randomly recruited during routine preventive examinations (annual physical examinations) at the Tianjin Medical University General Hospital-Health Management Centre, the largest and most comprehensive physical examination center in Tianjin. The protocol of the study was approved by the Institutional Review Board of Tianjin Medical University, and all of the participants gave written informed consent before participating in the study. The study conforms to the Strengthening the Reporting of Observational Studies in Epidemiology (STROBE) guidelines for cross-sectional and cohort studies.
TCLSIHealth data from 2007 to 2013 was used in the present study. All of the variables were measured at seven time points. The participant selection process is described in Fig. 1. During the research period, there were 101490 participants who had received at least one annual physical examination. We excluded participants who did not have leukocyte counts $(n=5914)$, fasting blood sugar (FBS) testing $(n=519)$, those with a history of CVD $(n=4231)$ or cancer $(n=589)$, those who had inflammatory diseases such as vasculitis, lupus, gastritis, chronic cholecystitis, nephritis, rhinitis, pharyngitis, bronchitis, myocarditis, atherosclerosis, arthritis, psoriasis, or trauma $(n=1472)$, and those who had a history of liver diseases $(n=888)$ or kidney diseases $(n=191)$. Owing to these exclusions, the final cross-sectional study population comprised 87686 participants.

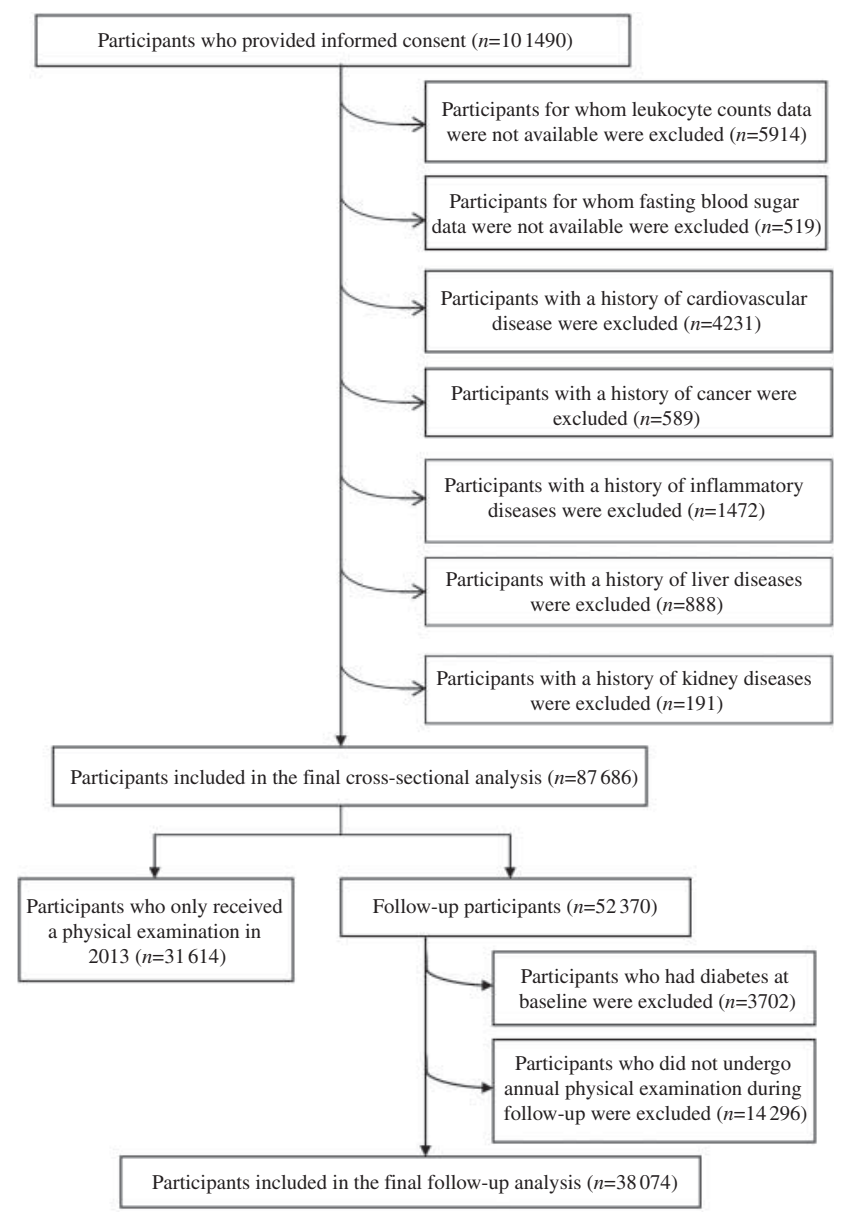

Figure 1

Selection of the study population. Tianjin Chronic Low-Grade Systemic Inflammation and Health (TCLSIHealth) Cohort Study, 2007 to 2013. 
For follow-up analysis, participants were excluded at baseline if they had received an annual physical examination only in $2013(n=31614)$ or had T2D $(n=3702)$. We also excluded 14296 participants who did not undergo health examinations during follow-up. Following these exclusions, the final cohort study population comprised 38074 participants (follow-up rate: $72.7 \%$ ). Incidence of T2D was evaluated across the $\sim 6$-year follow-up period (median follow-up 2.7 years).

\section{Assessment of T2D}

FBS was measured by the glucose oxidase method using reagents from Roche Diagnostics on an automatic biochemistry analyzer (Roche Cobas 8000 modular analyzer). T2D was defined in accordance with the criteria of the World Health Organization (15). Participants were considered to have T2D when their FBS level was $\geq 7 \mathrm{mmol} / \mathrm{l}$ or when they had physician-diagnosed diabetes and/or were currently using antidiabetic medications. We assigned the date of onset of T2D as the midpoint between the examination date when diabetes was first noted and the previous examination date. All of the participants were followed from the baseline examination to the date of incident diabetes or, for those with no diabetes, through the final examination date in 2013.

\section{Assessment of NLR}

Leukocyte, neutrophil, and lymphocyte counts were carried out using the automated hematology analyzer and expressed as $\times 1000$ cells $/ \mathrm{mm}^{3}$. The test for blanks was $\leq 0.2 \times 10^{9}$ cells $/ 1$. Then, by dividing the neutrophil by the lymphocyte count, we calculated the NLR. In order to investigate how the NLR, leukocyte, neutrophil, and lymphocyte counts are related to the prevalence and incidence of T2D, we divided them into five categories according to quintiles of participants.

\section{Assessment of other variables}

Blood pressure (BP) was measured twice from the upper left arm using an automatic device (Andon, Tianjin, China) after 5 min of rest in a seated position. The mean of these two measurements was taken as the BP value. Hypertension was defined as having a systolic BP (SBP) of $\geq 140 \mathrm{mmHg}$ and/or a diastolic BP (DBP) of $\geq 90 \mathrm{mmHg}$, a history of hypertension, or the current use of antihypertensive medications. Blood samples for the analysis of lipids were collected in siliconized vacuum lastics tubes.
Total cholesterol (TC) and triglycerides (TG) were measured by enzymatic methods, LDL was measured by the polyvinyl sulfuric acid precipitation method, and HDL was measured by the chemical precipitation method using reagents from Roche Diagnostics on an automatic biochemistry analyzer (Roche Cobas 8000 modular analyzer). Hyperlipidemia was defined as having a TC of $\geq 5.17 \mathrm{mmol} / 1$, a TG of $\geq 1.7 \mathrm{mmol} / 1$, an LDL of $\geq 3.37 \mathrm{mmol} / \mathrm{l}$, a history of hyperlipidemia, or the current use of antihyperlipidemic medications.

Anthropometric parameters (height and body weight) were recorded using a standard protocol. BMI was calculated as weight $/$ height ${ }^{2}\left(\mathrm{~kg} / \mathrm{m}^{2}\right)$. Waist circumference (WC) was measured at the umbilical level with participants standing and breathing normally. Visceral adiposity index (VAI) score was calculated as described using the following sex-specific equations (16):

Males :

$\mathrm{VAI}=\left(\frac{\mathrm{WC}}{39.68+1.88 \times \mathrm{BMI}}\right) \times\left(\frac{\mathrm{TG}}{1.03}\right) \times\left(\frac{1.31}{\mathrm{HDL}}\right) \quad$ and

Females :

$\mathrm{VAI}=\left(\frac{\mathrm{WC}}{36.58+1.89 \times \mathrm{BMI}}\right) \times\left(\frac{\mathrm{TG}}{0.81}\right) \times\left(\frac{1.52}{\mathrm{HDL}}\right)$.

Socio-demographic variables, including sex and age, were also assessed. A detailed personal and family history of physical illness and current medications was noted from 'yes' or 'no' responses to relevant questions. Information on alcohol and tobacco use was obtained from a questionnaire survey.

\section{Statistical analysis}

All statistical analyses were performed using the Statistical Analysis System 9.3 edition for Windows (SAS Institute, Inc., Cary, NC, USA). Because all of the continuous variables were non-normally distributed, they were logarithmically transformed to obtain substantially normal distributions before analysis, and the geometric means (95\% CI) are shown. For further analysis, the prevalence and incidence of T2D was used as a dependent variable, and the quintiles of NLR, leukocyte, neutrophil, and lymphocyte counts were used as independent variables. For baseline characteristics analysis, the differences among quintiles of NLR were examined using ANOVA for continuous variables and logistic regression analysis for proportional variables. Bonferroni-corrected $P$ values were used for comparisons between NLR quintiles. 
For cross-sectional analysis, multiple logistic regression analysis was used to examine the relationship between quintiles of NLR and the prevalence of T2D after adjusting for covariates, including age, sex, BMI, smoking status, drinking status, hypertension, hyperlipidemia, and family history of CVD, hypertension, hyperlipidemia, or diabetes; odds ratios (ORs) (95\% CI) were calculated. For follow-up analysis, Cox proportional hazards regression model was used (after adjusting for the baseline covariates just mentioned) to examine the relationships between quintiles of baseline NLR, the mean of annual NLR measurements (mean NLR), leukocyte, neutrophil, lymphocyte counts, and the incidence of T2D; hazard ratios (HRs) (95\% CI) were calculated. Moreover, the interaction between quintiles of NLR and sex was tested by the addition of this cross-product term to the regression model. All $P$ values for linear trends were calculated using the median value of quintiles of NLR. A Pearson's correlation coefficient $(r)$ was calculated to evaluate the relationship between mean and baseline NLR. All tests were two-tailed, and $P<0.05$ was defined as statistically significant.

\section{Results}

In the cross-sectional analysis, 48191 of the 87686 participants $(55.0 \%)$ were males. Overall mean \pm s.D. age was $40.1 \pm 13.3$ years. The overall prevalence of T2D was $4.9 \%$ (4413 of 87686 ). The characteristics of participants across NLR quintiles for cross-sectional analysis are presented in Table 1. Compared to participants in the lowest quintile of NLR, participants in the upper four quintiles tended to be older, to have higher BMI, waist circumference, and levels of TG, and to have a higher SBP, DBP, and FBS but a lower TC, LDL, and HDL; in addition, a higher proportion these participants were current smokers and drinkers and had a family history of CVD, hypertension, and diabetes ( $P$ for trends $<0.05$ ). Other than these results, no significant differences were observed between the participants in different NLR quintiles. Table 2 shows the crude and adjusted relationships between quintiles of NLR and T2D. In the final multivariate models, the ORs (95\% CI) for T2D across NLR quintiles were 1.00 (reference), 1.16 (1.04, 1.29), 1.22 (1.09, 1.36), 1.23 $(1.11,1.37)$, and $1.34(1.21,1.49)(P$ for trend $<0.0001)$.

Table 1 Participant characteristics by quintiles of neutrophil:lymphocyte ratio $(n=87686)$.

\begin{tabular}{|c|c|c|c|c|c|c|}
\hline \multirow{3}{*}{$\begin{array}{l}\text { Participant } \\
\text { characteristics }\end{array}$} & \multicolumn{5}{|c|}{ Quintiles of neutrophil:lymphocyte ratio (range) } & \multirow{3}{*}{$\begin{array}{l}P \text { for } \\
\text { trend }^{\mathrm{a}}\end{array}$} \\
\hline & \multirow{2}{*}{$\begin{array}{c}\text { Level } 1(0.11-1.21) \\
\quad(n=17542)\end{array}$} & \multirow{2}{*}{$\begin{array}{c}\text { Level } 2(1.21-1.48) \\
(n=17539)\end{array}$} & \multirow{2}{*}{$\begin{array}{c}\text { Level } 3(1.48-1.77) \\
(n=17472)\end{array}$} & \multirow{2}{*}{$\begin{array}{c}\text { Level } 4(1.77-2.17) \\
(n=17585)\end{array}$} & \multirow{2}{*}{$\frac{\text { Level } 5(2.17-17.20)}{(n=17547)}$} & \\
\hline & & & & & & \\
\hline Age $\left(\right.$ years) ${ }^{\mathrm{b}}$ & $38.7(38.5,38.9)^{b}$ & $39.2(39.0,39.4)$ & $39.8(39.6,40.0)$ & $40.6(40.4,40.8)$ & $42(41.8,42.2)$ & $<0.0001$ \\
\hline Sex (males, \%) & 53.4 & 55.8 & 56.5 & 55.6 & 53.5 & 0.24 \\
\hline BMI $\left(\mathrm{kg} / \mathrm{m}^{2}\right)^{\mathrm{b}}$ & $24.0(24.0,24.1)$ & $24.5(24.4,24.5)$ & $24.7(24.6,24.8)$ & $24.8(24.7,24.8)$ & $24.6(24.6,24.7)$ & $<0.0001$ \\
\hline $\begin{array}{l}\text { Waist circumference } \\
(\mathrm{cm})^{\mathrm{b}}\end{array}$ & $80.9(80.8,81.1)$ & $82.1(81.9,82.3)$ & $82.9(82.7,83)$ & $83.1(83.0,83.3)$ & $82.9(82.7,83.1)$ & $<0.0001$ \\
\hline $\mathrm{TC}(\mathrm{mmol} / \mathrm{l})^{\mathrm{c}}$ & $5.02(5.00,5.03)$ & $5.01(5.00,5.03)$ & $4.98(4.96,4.99)$ & $4.97(4.96,4.99)$ & $4.92(4.90,4.93)$ & $<0.0001$ \\
\hline TG $(\mathrm{mmol} / \mathrm{l})^{\mathrm{c}}$ & $1.43(1.41,1.45)$ & $1.50(1.48,1.53)$ & $1.53(1.51,1.55)$ & $1.55(1.53,1.57)$ & $1.48(1.46,1.50)$ & $<0.0001$ \\
\hline LDL $(\mathrm{mmol} / \mathrm{l})^{\mathrm{c}}$ & $3.08(3.07,3.10)$ & $3.05(3.04,3.07)$ & $3.02(3.01,3.04)$ & $3.01(3.00,3.03)$ & $2.96(2.94,2.98)$ & $<0.0001$ \\
\hline $\mathrm{HDL}(\mathrm{mmol} / \mathrm{l})^{\mathrm{c}}$ & $1.45(1.44,1.45)$ & $1.41(1.41,1.42)$ & $1.41(1.40,1.41)$ & $1.40(1.39,1.40)$ & $1.40(1.39,1.40)$ & $<0.0001$ \\
\hline SBP $(m m H g)^{b}$ & $120.3(120.0,120.5)$ & $120.9(120.6,121.1)$ & $121.4(121.2,121.7)$ & $121.8(121.6,122.1)$ & $122.5(122.2,122.8)$ & $<0.0001$ \\
\hline $\mathrm{DBP}(\mathrm{mmHg})^{\mathrm{b}}$ & $76.1(75.9,76.2)$ & $76.8(76.7,77.0)$ & $77.3(77.1,77.4)$ & $77.5(77.3,77.7)$ & $77.7(77.5,77.9)$ & $<0.0001$ \\
\hline FBS $(\mathrm{mmol} / \mathrm{l})^{\mathrm{b}}$ & $4.90(4.88,4.91)$ & $4.94(4.92,4.95)$ & $4.97(4.96,4.99)$ & $4.99(4.97,5.01)$ & $5.03(5.01,5.04)$ & $<0.0001$ \\
\hline VAI & $1.79(1.75,1.83)$ & $1.89(1.85,1.93)$ & $1.90(1.86,1.94)$ & $1.95(1.91,1.99)$ & $1.85(1.82,1.89)$ & $<0.0001$ \\
\hline \multicolumn{7}{|l|}{ Smoking status (\%) } \\
\hline Smoker & 22.6 & 25.3 & 26.3 & 27.8 & 27.4 & $<0.0001$ \\
\hline Ex-smoker & 0.03 & 0.04 & 0.02 & 0.05 & 0.04 & 0.65 \\
\hline Drinker (\%) & 34.5 & 37.1 & 38.5 & 37.8 & 36.8 & $<0.0001$ \\
\hline \multicolumn{7}{|l|}{$\begin{array}{c}\text { Family history of } \\
\text { diseases (\%) }\end{array}$} \\
\hline CVD & 21.1 & 22.7 & 23.4 & 23.3 & 23.3 & $<0.0001$ \\
\hline Hypertension & 37.9 & 39.7 & 40.2 & 40.8 & 40.0 & $<0.0001$ \\
\hline Hyperlipidemia & 0.50 & 0.56 & 0.61 & 0.52 & 0.57 & 0.52 \\
\hline Diabetes & 15.0 & 15.6 & 16.7 & 16.7 & 16.2 & $<0.001$ \\
\hline
\end{tabular}

TC, total cholesterol; TG, triglycerides; SBP, systolic BP; DBP, diastolic BP; FBS, fasting blood sugar; VAI, visceral adiposity index; CVD, cardiovascular disease. aNOVA or logistic regression analysis.

bMean $(95 \% \mathrm{Cl})$.

${ }^{\mathrm{C}} \mathrm{Geometric}$ mean $(95 \% \mathrm{Cl})$. 
Table 2 Adjusted relationships of quintiles of neutrophil:lymphocyte ratio to type 2 diabetes $(n=87686)$.

\begin{tabular}{|c|c|c|c|c|c|c|}
\hline \multirow[b]{3}{*}{ Adjusted relationships } & \multicolumn{5}{|c|}{ Quintiles of neutrophil:lymphocyte ratio (range) } & \multirow{3}{*}{$\begin{array}{l}P \text { for } \\
\text { trend }^{a}\end{array}$} \\
\hline & Level $1(0.11-1.21)$ & Level 2 (1.21-1.48) & Level 3 (1.48-1.77) & Level 4 (1.77-2.17) & Level 5 (2.17-17.20) & \\
\hline & $(n=17539)$ & $(n=17472)$ & $(n=17585)$ & $(n=17547)$ & $(n=17539)$ & \\
\hline $\begin{array}{l}\text { Number of type } 2 \text { diabetes } \\
\text { (fasting blood glucose } \\
\geq 7.0 \mathrm{mmol} / \mathrm{l})\end{array}$ & 664 & 807 & 886 & 956 & 1100 & - \\
\hline Crude & Reference & $1.23(1.10,1.36)^{b}$ & $1.36(1.23,1.51)$ & $1.46(1.32,1.62)$ & $1.70(1.54,1.88)$ & $<0.0001$ \\
\hline $\begin{array}{l}\text { Age-, sex-, and } \\
\text { BMI-adjusted }\end{array}$ & Reference & $1.17(1.05,1.30)$ & $1.23(1.11,1.37)$ & $1.25(1.12,1.38)$ & $1.33(1.20,1.47)$ & $<0.0001$ \\
\hline Multiple adjusted $^{\mathrm{C}}$ & Reference & $1.16(1.04,1.29)$ & $1.22(1.09,1.36)$ & $1.23(1.11,1.37)$ & $1.34(1.21,1.49)$ & $<0.0001$ \\
\hline
\end{tabular}

Similar relationships were also observed when males and females were analyzed separately ( $P$ for interaction 0.14 ).

In the cohort analysis, of the 38074 participants, 20134 (52.9\%) were males. Overall mean \pm s.D. age was $38.0 \pm 12.7$ years. The baseline characteristics for follow-up analysis are shown in Table 3. The baseline results were similar to the participant characteristics in the cross-sectional analysis, with the exception of FBS and the proportion of males. No significant differences in the levels of FBS, proportion of exsmokers or family history of hyperlipidemia were observed across NLR quintiles ( $P$ for trend $=0.40,0.80$ and 0.39 , respectively). During this period, a total of 691 participants received a new diagnosis of $\mathrm{T} 2 \mathrm{D}$. The incidence of $\mathrm{T} 2 \mathrm{D}$ was 6.84 per 1000 person-years. During the follow-up, incidences of T2D were 5.42, 6.25, 6.41, 7.49, and 9.01 per 1000 person-years respectively with the progression of NLR quintiles. The crude and adjusted relationships between NLR and the incidence of T2D are indicated in Table 4 . In the crude model, the unadjusted HRs (95\% CI) of T2D were related to a gradual increase in the levels of NLR as compared to participants who had the lowest quintile of NLR, and they were as follows: $1.12(0.86,1.44), 1.11(0.85,1.43), 1.39$ $(1.08,1.78)$, and $1.66(1.30,2.11)$ respectively ( $P$ for trend $<0.001)$. In the final multivariate models, the HRs (95\% CI) of T2D were related to a gradual increase in NLR levels as compared to participants with the lowest quintile of NLR, and they were as follows: $1.00(0.77,1.30), 0.99(0.77,1.28)$, $1.17(0.91,1.50)$, and $1.39(1.09,1.78)$ respectively $(P$ for trend $<0.01)$. Similar relationships were also observed when males and females were analyzed separately ( $P$ for interaction 0.998). The present study was based on single measurements of blood NLR, which may not reflect inflammatory status over time, so we further evaluated the relationship between mean NLR and the incidence of T2D as well as the relationship between mean and baseline NLR. After multiple adjustments, the HRs (95\% CI) of T2D were related to a gradual increase in mean NLR levels as compared to participants with the lowest quintile of mean NLR, and they were as follows: $1.08(0.84,1.41), 0.89(0.68,1.16)$, $1.17(0.91,1.51)$, and $1.38(1.08,1.76)$ respectively $(P$ for trend $<0.01)$. Mean NLR was positively and significantly correlated with baseline NLR by Pearson's correlation coefficient analysis $(r=0.924, P<0.0001)$.

Moreover, we divided leukocyte, neutrophil, and lymphocyte counts into five categories according to quintiles of participants as follows (range $\times 1000$ cells $/ \mathrm{mm}^{3}$ ): 1.10-4.60, 4.61-5.30, 5.31-5.91, 6.00-6.71, and $6.80-15.91 ; 0.10-2.40,2.40-2.90,2.90-3.39,3.39$ 4.00 , and 4.00-13.00; 0.40-1.60, 1.61-1.80, 1.81-2.01, 2.10-2.40, and 2.41-8.40 respectively. After adjusting for potential confounders, the HRs $(95 \% \mathrm{CI})$ of $\mathrm{T} 2 \mathrm{D}$ for increasing quintiles of leukocyte, neutrophil, and lymphocyte counts were 1.00, $0.88(0.66,1.19), 0.95(0.71$, $1.27), 1.18(0.90,1.56)$, and $1.22(0.94,1.60) ; 1.00,0.78$ (0.57, 1.06), $1.01(0.76,1.34), 1.07(0.81,1.40)$, and 1.28 $(0.99,1.67)$; and 1.00, $0.90(0.68,1.20), 1.01(0.78,1.32)$, $1.14(0.90,1.44)$, and $0.99(0.78,1.26)$ respectively (Fig. 2).

\section{Discussion}

In the present study, NLR, but not leukocyte, neutrophil, and lymphocyte counts, was positively related to the incidence of T2D in a reasonably sized sample of urban Chinese adults. This was a large-scale comprehensive study to relate the risk of T2D to NLR, an inflammatory biomarker that is used extensively in the medical field.

To date, only one (5-year) cohort study has assessed the relationship between differential leukocyte counts and 
Table 3 Participant characteristics by quintiles of neutrophil:lymphocyte ratio $(n=38074)$.

\begin{tabular}{|c|c|c|c|c|c|c|}
\hline \multirow{3}{*}{$\begin{array}{l}\text { Participant } \\
\text { characteristics }\end{array}$} & \multicolumn{5}{|c|}{ Quintiles of neutrophil:lymphocyte ratio (range) } & \multirow{3}{*}{$\begin{array}{l}P \text { for } \\
\text { trend }^{\mathrm{a}}\end{array}$} \\
\hline & Level $1(0.11-1.22)$ & Level 2 (1.22-1.48) & Level 3 (1.48-1.77) & Level 4 (1.77-2.18) & Level 5 (2.18-17.20) & \\
\hline & $(n=8325)$ & $(n=7782)$ & $(n=7714)$ & $(n=7369)$ & $(n=6884)$ & \\
\hline Age (years) ${ }^{b}$ & $36.7(36.5,37)^{b}$ & $37.1(36.9,37.4)$ & $37.9(37.6,38.2)$ & $38.7(38.4,39.0)$ & $39.8(39.5,40.1)$ & $<0.0001$ \\
\hline Sex (males, \%) & 52.7 & 53.6 & 55.0 & 53.0 & 50.0 & $<0.01$ \\
\hline BMI $\left(\mathrm{kg} / \mathrm{m}^{2}\right)^{\mathrm{b}}$ & $23.8(23.8,23.9)$ & $24.2(24.1,24.2)$ & $24.3(24.3,24.4)$ & $24.5(24.4,24.5)$ & $24.3(24.2,24.4)$ & $<0.0001$ \\
\hline $\begin{array}{l}\text { Waist circumference } \\
(\mathrm{cm})^{\mathrm{b}}\end{array}$ & $80.0(79.7,80.2)$ & $80.7(80.4,80.9)$ & $81.5(81.2,81.7)$ & $81.7(81.5,82.0)$ & $81.5(81.2,81.7)$ & $<0.0001$ \\
\hline $\mathrm{TC}(\mathrm{mmol} / \mathrm{l})^{\mathrm{C}}$ & $5.00(4.98,5.02)$ & $4.98(4.96,5.00)$ & $4.95(4.93,4.97)$ & $4.95(4.93,4.97)$ & $4.90(4.88,4.92)$ & $<0.01$ \\
\hline TG $(\mathrm{mmol} / \mathrm{l})^{\mathrm{c}}$ & $1.35(1.32,1.38)$ & $1.39(1.37,1.42)$ & $1.42(1.39,1.45)$ & $1.43(1.41,1.46)$ & $1.38(1.35,1.41)$ & $<0.001$ \\
\hline LDL $(\mathrm{mmol} / \mathrm{l})^{c}$ & $3.12(3.09,3.14)$ & $3.07(3.05,3.09)$ & $3.04(3.02,3.06)$ & $3.02(3.00,3.04)$ & $2.97(2.95,3.00)$ & $<0.0001$ \\
\hline $\mathrm{HDL}(\mathrm{mmol} / \mathrm{l})^{c}$ & $1.45(1.44,1.46)$ & $1.42(1.41,1.43)$ & $1.41(1.40,1.42)$ & $1.40(1.39,1.41)$ & $1.41(1.40,1.42)$ & $<0.0001$ \\
\hline $\mathrm{SBP}(\mathrm{mmHg})^{\mathrm{b}}$ & $120.5(120.2,120.9)$ & $121.1(120.8,121.5)$ & $121.6(121.2,122.0)$ & $121.9(121.5,122.2)$ & $122.6(122.2,123.0)$ & $<0.0001$ \\
\hline $\mathrm{DBP}(\mathrm{mmHg})^{\mathrm{b}}$ & $76.0(75.8,76.3)$ & $76.9(76.6,77.1)$ & $77.2(77.0,77.5)$ & $77.4(77.1,77.6)$ & $77.8(77.5,78.0)$ & $<0.0001$ \\
\hline FBS $(\mathrm{mmol} / \mathrm{l})^{\mathrm{b}}$ & $4.74(4.73,4.76)$ & $4.75(4.73,4.76)$ & $4.75(4.74,4.76)$ & $4.76(4.75,4.78)$ & $4.75(4.74,4.77)$ & 0.4 \\
\hline VAI & $1.67(1.62,1.72)$ & $1.72(1.67,1.77)$ & $1.76(1.71,1.81)$ & $1.80(1.75,1.85)$ & $1.70(1.65,1.75)$ & $<0.01$ \\
\hline \multicolumn{7}{|l|}{ Smoking status (\%) } \\
\hline Smoker & 20.0 & 22.3 & 24.1 & 24.5 & 24.0 & $<0.0001$ \\
\hline Ex-smoker & 0.02 & 0.04 & 0.01 & 0.04 & 0.01 & 0.80 \\
\hline Drinker (\%) & 29.3 & 31.7 & 33.9 & 33.0 & 31.7 & $<0.0001$ \\
\hline \multicolumn{7}{|l|}{$\begin{array}{c}\text { Family history of } \\
\text { diseases (\%) }\end{array}$} \\
\hline CVD & 27.1 & 29.5 & 30.3 & 30.3 & 31.4 & $<0.0001$ \\
\hline Hypertension & 45.8 & 47.9 & 47.9 & 49.4 & 49.7 & $<0.0001$ \\
\hline Hyperlipidemia & 0.74 & 0.99 & 0.92 & 0.87 & 0.94 & 0.39 \\
\hline Diabetes & 17.6 & 18.2 & 19.0 & 19.4 & 19.2 & $<0.01$ \\
\hline
\end{tabular}

TC, total cholesterol; TG, triglycerides; SBP, systolic BP; DBP, diastolic BP; FBS, fasting blood sugar; VAl, visceral adiposity index; CVD, cardiovascular disease ${ }^{a}$ ANOVA or logistic regression analysis.

${ }^{\mathrm{b}}$ Mean $(95 \% \mathrm{Cl})$.

'Geometric mean $(95 \% \mathrm{Cl})$.

the incidence of T2D, and it did so in a small middle-aged population ( $n=866$; number of new diabetes cases: 138 ) (11). Interestingly, in that study, the tertiles of lymphocyte-only count (but not leukocyte, neutrophil, or monocyte counts or NLR) were independently and significantly related to the incidence of T2D. Furthermore, a small-scale cross-sectional study by Lee et al. (17) claimed that NLR was not associated with $\beta$-cell dysfunction and that the association between NLR and insulin resistance was confounded by obesity. Although the reasons for this discrepancy remain unclear, we speculate that the smaller sample size and substantial number of new diabetes cases in that study might have blurred the relationship between differential leukocyte counts and the incidence of T2D. Further studies are required to evaluate whether NLR and/or lymphocyte count is a useful marker for predicting the incidence of T2D.

T2D seems to result - at least initially - from an activation of the innate immune system in the organs that are involved in glucose metabolism (18). Several studies have suggested that there might be a link between inflammatory markers and diabetes. Cross-sectional studies in nondiabetic subjects or the general population
$(19,20,21)$ and those in individuals with impaired glucose tolerance/impaired fasting glucose $(22,23,24)$ have shown that acute-phase reactants such as CRP, IL6, and tumor necrosis factor $\alpha(\mathrm{TNF} \alpha)$ are positively correlated with insulin resistance and plasma insulin concentration. In one study by Leinonen et al. (25), all of the markers of inflammation, including CRP, serum amyloid A, secretory phospholipase A2, and IL6, as well as soluble cell adhesion molecules correlated with homeostasis model-measured insulin resistance. Together, this evidence suggests that inflammation participates in the pathogenesis of T2D (3). Moreover, previous works have also indicated that inflammation markers might be accurate predictors of T2D. Using data from the Atherosclerosis Risk in Communities Study, Schmidt et al. (26) were the first to show that a variety of inflammatory markers, including leukocyte count and low serum albumin, $\alpha 1$-acid glycoprotein, fibrinogen, and sialic acid, accurately predict T2D development in a middle-aged population. This finding has been supported by other studies that showed the same correlation in both adult males and females across several populations $(7,27,28,29)$. These findings suggest that inflammation makers are useful predictors of T2D. 
Table 4 Cohort analysis: adjusted relationships of quartiles of baseline neutrophil:lymphocyte ratio to the incidence of type 2 diabetes $(n=38$ 074).

\begin{tabular}{|c|c|c|c|c|c|c|}
\hline \multirow[b]{3}{*}{ Adjusted relationships } & \multicolumn{5}{|c|}{ Quintiles of neutrophil:lymphocyte ratio (range) } & \multirow{3}{*}{$\begin{array}{l}P \text { for } \\
\text { trend }^{\mathrm{a}}\end{array}$} \\
\hline & Level $1(0.11-1.22)$ & Level $2(1.22-1.48)$ & Level 3 (1.48-1.77) & Level 4 (1.77-2.18) & Level $5(2.18-17.20)$ & \\
\hline & $(n=8325)$ & $(n=7782)$ & $(n=7714)$ & $(n=7369)$ & $(n=6884)$ & \\
\hline Person-years of follow-up & 22127 & 20489 & 20756 & 19488 & 18196 & - \\
\hline Number of type 2 diabetes & 120 & 128 & 133 & 146 & 164 & - \\
\hline Crude & Reference & $1.12(0.86,1.44)^{b}$ & $1.11(0.85,1.43)$ & $1.39(1.08,1.78)$ & $1.66(1.30,2.11)$ & $<0.001$ \\
\hline Age-, sex-, and BMI-adjusted & Reference & $1.03(0.80,1.34)$ & $1.00(0.78,1.30)$ & $1.19(0.93,1.53)$ & $1.40(1.10,1.79)$ & $<0.01$ \\
\hline Multiple adjusted $^{c}$ & Reference & $1.00(0.77,1.30)$ & $0.99(0.77,1.28)$ & $1.17(0.91,1.50)$ & $1.39(1.09,1.78)$ & $<0.01$ \\
\hline
\end{tabular}

NLR reflects both the lymphocyte and neutrophil counts. The power of NLR as an inflammatory factor stems from both a reduction in the lymphocyte count and an increase in the neutrophil count. Tanaka et al. (30) showed that $\mathrm{T}$ lymphocytes were reduced in obese people and that lymphopenia appeared to be related to inflammation through TNF $\alpha$. Neutrophils are the first immune cells to respond to inflammation and can exacerbate the chronic inflammatory state by helping recruit macrophages and by interacting with antigen-presenting cells. An animal study demonstrated that neutrophil elastase could degrade the insulin receptor substrate 1 and reduce insulin-induced Akt phosphorylation in adipocytes. This mechanism may be involved in the neutrophil effect on insulin resistance (31). However, in the present results, only NLR (not lymphocyte, leukocyte, or neutrophil) was independently and significantly related to the incidence of T2D. Therefore, NLR may be a better biomarker of T2D than the others are.

Several studies have established the utility of NLR as a medically relevant biomarker. NLR can single out individuals that are at risk for sensorineural hearing loss (caused by vascular complications of diabetes via inflammation) (32) or those that are at risk for adverse cardiac events (33). One review article also indicated that NLR has been related to arterial stiffness and high coronary calcium scores, which are significant markers of CVD (34). Recently, NLR has been reported to be a prognostic marker for outcomes that result from diabetic retinopathy (35), including microvascular complications (36) and impaired renal function (37). Although the precise mechanisms that underlie the associations between systemic inflammation and prevalent conditions remain to be elucidated, these studies verify the present conclusion that NLR could be used as an innovative and effective predictor for T2D.
Inflammation can be a cause of numerous diabetic complications that represent a complex set of phenomena that stretch beyond the field of inflammation proper. Corvera et al. (38) proposed that during diabetic complications, the early formation of advanced glycation end products associated with hyperglycemia stimulates mechanisms that lead to the recruitment of key components of the inflammatory response. Indeed, studies have shown that the advanced glycation end products receptor-mediated regulation of adiposity and inflammation may result in T2D and diabetic vascular complications (39). Other research has shown that serum levels of IL6, IL17, interferon $\gamma$, TNF $\gamma$, IL2, and IL10 were increased in T2D nephropathy (T2DN)

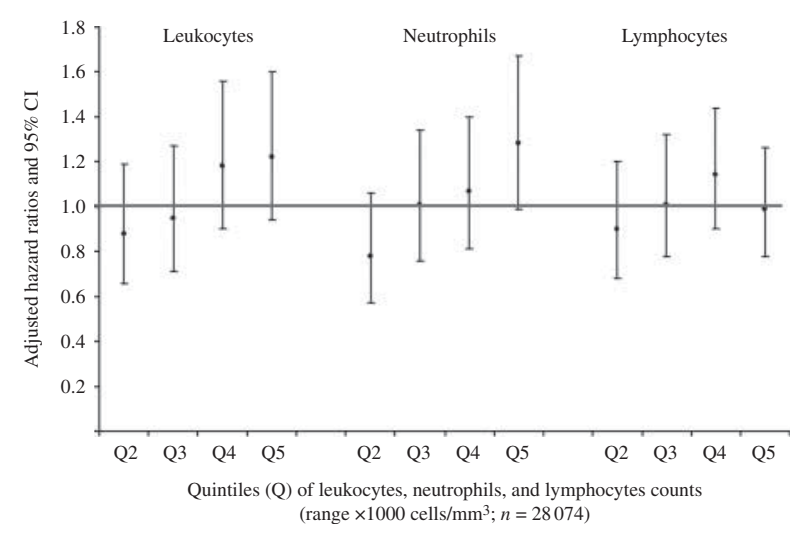

Figure 2

Adjusted hazard ratios (HRs) $(95 \% \mathrm{Cl})$ of the relationship between the quintiles (Q) of leukocyte, neutrophil, and lymphocyte counts and the incidence of type 2 diabetes. Adjusted for age, sex, BMI, smoking status, drinking status, hypertension, hyperlipidemia, and family history of cardiovascular disease, hypertension, hyperlipidemia, and diabetes. 
patients (40), and inflammatory responses (such as increased expression of toll-like receptors) were involved in the perpetuation of inflammation in the diabetic kidney (41). During the development and progression of T2DN, increased oxidative stress leads to the activation of the poly (ADP-ribose) polymerase pathway, which regulates the expression of genes that are involved in promoting inflammatory reactions (42). A study by Vinik et al. (43) emphasized that a loss of heart rate variability, which occurs early in the development of autonomic dysfunction, correlates with an increase in circulating inflammation markers, such as CRP and IL6. Collectively and combined with the present findings, these results suggest that inflammation likely contributes to the pathophysiology of and complications that result from T2D; thus, further studies aimed at investigating the mechanisms that underlie these contributions are warranted.

Limitations of the present study should be noted. Even though the present study adjusted for a considerable number of potential confounding factors, we cannot exclude the possibility that NLR is affected by a lifestyle variable that was not collected by the TCLSIHealth study. Furthermore, the ascertainment of T2D by a single FBS might have impacted the definition of T2D and have had some influences on the analysis results.

\section{Conclusion}

In the present study, increased NLR levels were associated with the prevalence and incidence of T2D among the adult population. Our findings indicate that baseline NLR might be a useful predictive factor for T2D in the general adult population.

\section{Declaration of interest}

The authors declare that there is no conflict of interest that could be perceived as prejudicing the impartiality of the research reported.

\section{Funding}

This study was supported by grants from the Key Technologies R\&D Program of Tianjin (key project numbers 11ZCGYSY05700, 12ZCZDSY20400, and 13ZCZDSY20200), the Technologies Development Program of the Beichen District of Tianjin (grant numbers bcws2013-21 and bcws2014-05), the Technologies Project of the Tianjin Binhai New Area (grant numbers 2013-02-04 and 2013-02-06), and the Science Foundation of Tianjin Medical University (grant number 2010KY28).

Author contribution statement

$\mathrm{K}$ Niu conceived and designed the study. All of the authors contributed to the acquisition of the participants and data. K Niu, X Guo, and S Zhang analyzed the data and wrote the manuscript. K Niu, Q Zhang, K Song, and L Liu contributed to supervision. C Wang, H Shi, Y Xia, X Liu, C Li, S Sun, $X$ Wang, M Zhou, G Huang, H Zhao, and Q Jia contributed to discussion and edited the manuscript.

\section{Acknowledgements}

We gratefully acknowledge all of the men and women who participated in the study and the Tianjin Medical University General Hospital-Health Management Centre for creating the possibility to perform the study.

\section{References}

1 Shaw JE, Sicree RA \& Zimmet PZ. Global estimates of the prevalence of diabetes for 2010 and 2030. Diabetes Research and Clinical Practice 2010 87 4-14. (doi:10.1016/j.diabres.2009.10.007)

2 Yang W, Lu J, Weng J, Jia W, Ji L, Xiao J, Shan Z, Liu J, Tian H, Ji Q et al. Prevalence of diabetes among men and women in China. New England Journal of Medicine 2010362 1090-1101. (doi:10.1056/NEJMoa0908292)

3 Schmidt MI, Duncan BB, Bang H, Pankow JS, Ballantyne CM, Golden SH, Folsom AR \& Chambless LE. Identifying individuals at high risk for diabetes: the Atherosclerosis Risk in Communities study. Diabetes Care 200528 2013-2018. (doi:10.2337/diacare.28.8.2013)

4 Levitzky YS, Pencina MJ, D'Agostino RB, Meigs JB, Murabito JM, Vasan RS \& Fox CS. Impact of impaired fasting glucose on cardiovascular disease: the Framingham Heart Study. Journal of American College of Cardiology 200851 264-270. (doi:10.1016/j.jacc.2007.09.038)

5 Li R, Lu W, Jiang QW, Li YY, Zhao GM, Shi L, Yang QD, Ruan Y, Jiang J, Zhang SN et al. Increasing prevalence of type 2 diabetes in Chinese adults in Shanghai. Diabetes Care 201235 1028-1030. (doi:10.2337/dc11-1212)

6 Donath MY \& Shoelson SE. Type 2 diabetes as an inflammatory disease. Nature Reviews. Immunology 201111 98-107. (doi:10.1038/nri2925)

7 Pradhan AD, Manson JE, Rifai N, Buring JE \& Ridker PM. C-reactive protein, interleukin 6 , and risk of developing type 2 diabetes mellitus. JAMA 2001286 327-334. (doi:10.1001/jama.286.3.327)

8 Donath MY, Boni-Schnetzler M, Ellingsgaard H, Halban PA \& Ehses JA. Cytokine production by islets in health and diabetes: cellular origin, regulation and function. Trends in Endocrinology and Metabolism 201021 261-267. (doi:10.1016/j.tem.2009.12.010)

9 Avci A, Elnur A, Goksel A, Serdar F, Servet I, Atilla K, Mustafa TM, Cuneyt T, Yeliz G, Mustafa B et al. The relationship between neutrophil/lymphocyte ratio and calcific aortic stenosis. Echocardiography 201431 1031-1035. (doi:10.1111/echo.12534)

10 Farah R \& Khamisy-Farah R. Association of neutrophil to lymphocyte ratio with presence and severity of gastritis due to Helicobacter pylori infection. Journal of Clinical Laboratory Analysis 201428 219-223. (doi:10.1002/jcla.21669)

11 Lorenzo C, Hanley AJ \& Haffner SM. Differential white cell count and incident type 2 diabetes: the Insulin Resistance Atherosclerosis Study. Diabetologia 201457 83-92. (doi:10.1007/s00125-013-3080-0)

12 Song K, Du H, Zhang Q, Wang C, Guo Y, Wu H, Liu L, Jia Q, Wang X, Shi $\mathrm{H}$ et al. Serum immunoglobulin $\mathrm{M}$ concentration is positively related to metabolic syndrome in an adult population: Tianjin Chronic Low-Grade Systemic Inflammation and Health (TCLSIH) cohort study. PLoS ONE 20149 e88701. (doi:10.1371/journal.pone.0088701)

13 Yu B, He H, Zhang Q, Wu H, Du H, Liu L, Wang C, Shi H, Xia Y, Guo X et al. Soft drink consumption is associated with depressive symptoms among adults in China. Journal of Affective Disorders 2014 172C 422-427. (doi:10.1016/j.jad.2014.10.026)

14 Vander Weg MW, Peterson AL, Ebbert JO, Debon M, Klesges RC \& Haddock CK. Prevalence of alternative forms of tobacco use in a population of young adult military recruits. Addictive Behaviors 200833 69-82. (doi:10.1016/j.addbeh.2007.07.005) 
15 Alberti KG \& Zimmet PZ. Definition, diagnosis and classification of diabetes mellitus and its complications. Part 1: diagnosis and classification of diabetes mellitus provisional report of a WHO consultation. Diabetic Medicine 199815 539-553. (doi:10.1002/ (SICI) 1096-9136(199807)15:7 < 539::AID-DIA668 > 3.0.CO;2-S)

16 Amato MC, Giordano C, Galia M, Criscimanna A, Vitabile S, Midiri M, Galluzzo A \& AlkaMeSy Study G, . Visceral adiposity index: a reliable indicator of visceral fat function associated with cardiometabolic risk. Diabetes Care 201033 920-922. (doi:10.2337/dc09-1825)

17 Lee CT, Harris SB, Retnakaran R, Gerstein HC, Perkins BA, Zinman B \& Hanley AJ. White blood cell subtypes, insulin resistance and $\beta$-cell dysfunction in high-risk individuals - the PROMISE cohort. Clinical Endocrinology 201481 536-541. (doi:10.1111/cen.12390)

18 Esser N, Legrand-Poels S, Piette J, Scheen AJ \& Paquot N. Inflammation as a link between obesity, metabolic syndrome and type 2 diabetes. Diabetes Research and Clinical Practice 2014105 141-150. (doi:10.1016/ j.diabres.2014.04.006)

19 Festa A, D'Agostino R Jr, Howard G, Mykkanen L, Tracy RP \& Haffner SM. Chronic subclinical inflammation as part of the insulin resistance syndrome: the Insulin Resistance Atherosclerosis Study (IRAS). Circulation 2000102 42-47. (doi:10.1161/01.CIR.102.1.42)

20 Sakkinen PA, Wahl P, Cushman M, Lewis MR \& Tracy RP. Clustering of procoagulation, inflammation, and fibrinolysis variables with metabolic factors in insulin resistance syndrome. American Journal of Epidemiology 2000152 897-907. (doi:10.1093/aje/152.10.897)

21 Weyer C, Yudkin JS, Stehouwer CD, Schalkwijk CG, Pratley RE \& Tataranni PA. Humoral markers of inflammation and endothelial dysfunction in relation to adiposity and in vivo insulin action in Pima Indians. Atherosclerosis 2002161 233-242. (doi:10.1016/S0021-9150(01)00626-8)

22 Muller S, Martin S, Koenig W, Hanifi-Moghaddam P, Rathmann W, Haastert B, Giani G, Illig T, Thorand B \& Kolb H. Impaired glucose tolerance is associated with increased serum concentrations of interleukin 6 and co-regulated acute-phase proteins but not TNF- $\alpha$ or its receptors. Diabetologia 200245 805-812. (doi:10.1007/s00125-002-0829-2)

23 Temelkova-Kurktschiev T, Henkel E, Koehler C, Karrei K \& Hanefeld M. Subclinical inflammation in newly detected type II diabetes and impaired glucose tolerance. Diabetologia 200245 151. (doi:10.1007/s125-002-8256-1)

24 Marsland AL, McCaffery JM, Muldoon MF \& Manuck SB. Systemic inflammation and the metabolic syndrome among middle-aged community volunteers. Metabolism 201059 1801-1808. (doi:10.1016/j. metabol.2010.05.015)

25 Leinonen E, Hurt-Camejo E, Wiklund O, Hulten LM, Hiukka A \& Taskinen MR. Insulin resistance and adiposity correlate with acutephase reaction and soluble cell adhesion molecules in type 2 diabetes. Atherosclerosis 2003 166 387-394. (doi:10.1016/S0021-9150(02)00371-4)

26 Schmidt MI, Duncan BB, Sharrett AR, Lindberg G, Savage PJ, Offenbacher S, Azambuja MI, Tracy RP \& Heiss G. Markers of inflammation and prediction of diabetes mellitus in adults (Atherosclerosis Risk in Communities study): a cohort study. Lancet 1999353 1649-1652. (doi:10.1016/S0140-6736(99)01046-6)

27 Festa A, D'Agostino R Jr, Tracy RP \& Haffner SM. Elevated levels of acute-phase proteins and plasminogen activator inhibitor-1 predict the development of type 2 diabetes: the insulin resistance atherosclerosis study. Diabetes 200251 1131-1137. (doi:10.2337/diabetes.51.4.1131)

28 Nakanishi N, Yoshida H, Matsuo Y, Suzuki K \& Tatara K. White bloodcell count and the risk of impaired fasting glucose or Type II diabetes in middle-aged Japanese men. Diabetologia 200245 42-48. (doi:10.1007/ s125-002-8243-1)

29 Spranger J, Kroke A, Mohlig M, Hoffmann K, Bergmann MM, Ristow M, Boeing H \& Pfeiffer AF. Inflammatory cytokines and the risk to develop type 2 diabetes: results of the prospective population-based European Prospective Investigation into Cancer and Nutrition (EPIC)-Potsdam Study. Diabetes 200352 812-817. (doi:10.2337/diabetes.52.3.812)

30 Tanaka S, Isoda F, Ishihara Y, Kimura M \& Yamakawa T.

$\mathrm{T}$ lymphopaenia in relation to body mass index and TNF- $\alpha$ in human obesity: adequate weight reduction can be corrective. Clinical Endocrinology 200154 347-354. (doi:10.1111/j.1365-2265.2001. $1139 \mathrm{cn} 2155 . \mathrm{x})$

31 Talukdar S, Oh da Y, Bandyopadhyay G, Li D, Xu J, McNelis J, Lu M, Li P, Yan Q, Zhu Y et al. Neutrophils mediate insulin resistance in mice fed a high-fat diet through secreted elastase. Nature Medicine 201218 1407-1412. (doi:10.1038/nm.2885)

32 Sukhija R, Aronow WS, Sorbera C, Peterson SJ, Frishman WH \& Cohen M. Mortality, left ventricular ejection fraction, and prevalence of new left ventricular wall motion abnormality at long-term follow-up in patients with implantable cardioverter defibrillators treated with biventricular pacing versus right ventricular pacing. American Journal of Therapeutics 2007 14 328-330. (doi:10.1097/01.mjt.0000209686.44948.e8)

33 Azab B, Chainani V, Shah N \& McGinn JT. Neutrophil-lymphocyte ratio as a predictor of major adverse cardiac events among diabetic population: a 4-year follow-up study. Angiology 201364 456-465. (doi:10.1177/0003319712455216)

34 Bhat T, Teli S, Rijal J, Bhat H, Raza M, Khoueiry G, Meghani M, Akhtar M \& Costantino T. Neutrophil to lymphocyte ratio and cardiovascular diseases: a review. Expert Review of Cardiovascular Therapy 201311 55-59. (doi:10.1586/erc.12.159)

35 Ulu SM, Dogan M, Ahsen A, Altug A, Demir K, Acarturk G \& Inan S. Neutrophil-to-lymphocyte ratio as a quick and reliable predictive marker to diagnose the severity of diabetic retinopathy. Diabetes Technology \& Therapeutics 201315 942-947. (doi:10.1089/dia.2013.0097)

36 Ozturk ZA, Kuyumcu ME, Yesil Y, Savas E, Yildiz H, Kepekci Y \& Ariogul S. Is there a link between neutrophil-lymphocyte ratio and microvascular complications in geriatric diabetic patients? Journal of Endocrinological Investigation 201336 593-599.

37 Azab B, Daoud J, Naeem FB, Nasr R, Ross J, Ghimire P, Siddiqui A, Azzi N, Rihana N, Abdallah M et al. Neutrophil-to-lymphocyte ratio as a predictor of worsening renal function in diabetic patients (3-year follow-up study). Renal Failure 201234 571-576. (doi:10.3109/ 0886022X.2012.668741)

38 Corvera S, Burkart A, Kim JY, Christianson J, Wang Z \& Scherer PE. Keystone meeting summary: 'Adipogenesis, obesity, and inflammation' and 'Diabetes mellitus and the control of cellular energy metabolism,' January 21-26, 2006, Vancouver, Canada. Genes Development 200620 2193-2201. (doi:10.1101/gad.1447506)

39 Yamamoto Y \& Yamamoto H. RAGE-mediated inflammation, type 2 diabetes, and diabetic vascular complication. Frontiers in Endocrinology 20134 105. (doi:10.3389/fendo.2013.00105)

40 Zhang C, Xiao C, Wang P, Xu W, Zhang A, Li Q \& Xu X. The alteration of Th1/Th2/Th17/Treg paradigm in patients with type 2 diabetes mellitus: relationship with diabetic nephropathy. Human Immunology 201475 289-296. (doi:10.1016/j.humimm.2014.02.007)

41 Mudaliar H, Pollock C \& Panchapakesan U. Role of toll-like receptors in diabetic nephropathy. Clinical Science 2014126 685-694. (doi:10.1042/ CS20130267)

42 Edwards JL, Vincent AM, Cheng HT \& Feldman EL. Diabetic neuropathy: mechanisms to management. Pharmacology \& Therapeutics 2008120 1-34. (doi:10.1016/j.pharmthera.2008.05.005)

43 Vinik AI, Erbas T \& Casellini CM. Diabetic cardiac autonomic neuropathy, inflammation and cardiovascular disease. Journal of Diabetes Investigation 20134 4-18. (doi:10.1111/jdi.12042)
Received 14 February 2015

Revised version received 21 April 2015

Accepted 7 May 2015 